\title{
Theoretical study on the dynamical correlation in Ar focusing on $3 s$ excitation with outer-shell ionization due to electron impacts
}

\author{
Tsutomu Watanabe, ${ }^{1,2, *}$ Andrew T. Domondon, ${ }^{3,4, \dagger}$ and Xiao-Min Tong ${ }^{5,6, \$}$ \\ ${ }^{1}$ Institute for Laser Science, University of Electro-Communications, Chofugaoka, Chofu, Tokyo 182-8585, Japan \\ ${ }^{2}$ Department of Physics, Tokyo Metropolitan University, 1-1 Minami-Ohsawa, Hachioji, Tokyo 192-0364, Japan \\ ${ }^{3}$ International Christian University, Ohsawa, Mitaka-shi, Tokyo 181-8585, Japan \\ ${ }^{4}$ Committee on Conceptual and Historical Studies of Science, University of Chicago, Room 205, 1126 East 59th Street, \\ Chicago, Illinois 60637, USA \\ ${ }^{5}$ Institute of Materials Science, Graduate School of Pure and Applied Sciences, University of Tsukuba, 1-1-1 Tennodai, \\ Tsukuba, Ibaraki 305-8573, Japan \\ ${ }^{6}$ Center for Computational Sciences, University of Tsukuba, 1-1-1 Tennodai, Tsukuba, Ibaraki 305-8577, Japan
}

(Received 6 July 2009; published 16 October 2009)

\begin{abstract}
We investigate the energy-loss spectra of high-energy electrons incident on Ar atoms using linear density response theory with a self-interaction correction. The energy region of the spectra covers from 24.5 to $30.0 \mathrm{eV}$, where many $n s, n p$, and $n d$ autoionizing resonant states from the $3 s$ orbital of Ar exist with the continuum background from the $3 p$ orbital. We compare the calculated spectra to recent energy-loss spectra measurements and identify the observed autoionizing resonant states. We also discuss the dependence of the spectra on the scattering angles and their physical meanings.
\end{abstract}

DOI: 10.1103/PhysRevA.80.042709

PACS number(s): 34.80.Dp, 32.70.Cs, 31.15.es

\section{INTRODUCTION}

The problem of how to treat electron correlation when electronic transitions are induced in an atom or molecule by an external field remains an important topic in contemporary physics [1]. In particular, atomic photoabsorption spectra of excited discrete states embedded in an ionization continuum have been studied extensively. Such problems have been investigated using energy-loss spectra from charged particles impacting atoms or ions [2-6]. These spectra not only show optically allowed transitions but also optically forbidden ones. By varying the scattering angle of incident charged particles, one can obtain information concerning the socalled dynamic correlation for both types of transitions. In addition to these energy-loss spectra studies, the existence and physical significance of resonant discrete states in the continuum background have been studied using various theoretical tools [1,7-9], such as configuration interaction [10], hyperspherical coordinates [11], close-coupling methods [12], and $R$-matrix theory [13]. Here, we only cite the most recent papers for each respective method. For a more detailed list, we refer the reader to a paper by Yuan et al. [14]. We note that most studies discuss the resonant energy positions and resonance widths, but do not pay attention to energy-loss spectra and their momentum-transfer dependence on the charged particles. Two important exceptions are an experimental study of the energy-loss spectra of electron incident on a He target [15] and a theoretical study of the same system using $R$-matrix theory [14].

In this paper, we present a calculation for the energy-loss spectra of an electron incident on an argon atom as a func-

\footnotetext{
*tsutomu@gol.com

†domondon@icu.ac.jp

†tong.xiaomin.ga@u.tsukuba.ac.jp
}

tion of the scattering angle and the momentum transfer of the scattering electron. The method is based on linear density response theory using an optimized effective potential incorporating a self-interaction correction [16]. Argon, the target atom in the process we examined in this paper, has been a subject of a number of theoretical and experimental studies. Examples of the former include a study by Fano [1] on photoabsorption phenomena in $\mathrm{Ar}$ and an $R$-matrix theory calculation by Burke and Taylor [17] on the photoexcitation of the inner-valence $3 s$ electron of Ar. Examples of the latter include studies by Madden et al. [6], Mitchell et al. [18], and Wu et al. [19]. Madden et al. [6] were the first to measure the photoabsorption spectra of Ar. Mitchell et al. $[18,20]$ measured the energy-loss spectra for the first time on $\mathrm{Ar}$ and $\mathrm{Ne}$ targets using electron energy-loss methods. Wu et al. [19] measured the spectra of $\mathrm{Ar}$ in the excitation energy region around $25-31 \mathrm{eV}$ and its momentum-transfer dependence. In this energy regime, excitations occur from the inner-shell $3 \mathrm{~s}$ orbital to $n p, n d$, and $n s$ Rydberg states up to the ionization limit of the $3 s 3 p^{6}{ }^{2} S$ state. Most recently, a Japan-China joint experimental group [21] measured the energy-loss spectra and obtained results compatible to those of Wu et al. Since these most recent experimental results cover a wide range of the momentum transfers or scattering angles, we compare our theoretical results to them and identify the autoionization states for both optically allowed and optically forbidden transitions.

\section{THEORETICAL METHOD}

Linear response theory [22-24] can be applied to various physical phenomena. The electron density-functional approach has extensively been used to study the dynamic correlation induced by photoionization processes. It takes the simplicity of the independent-particle approximation (IPA), while taking into account dynamic correlation effects. In an 
earlier paper [16], we applied linear density response theory to experiments involving autoionizing resonances in Ar induced by electron-impact ionization with small scattering angles (less than $3^{\circ}$ ). In this paper, we extend our research to a wide range of scattering angles and compare our results to recent experimental measurements. Since we provide a detailed explanation of the method in our earlier paper [16], we discuss it only briefly here.

According to linear density response theory, the frequency-dependent electron-density change $\delta \rho(\mathbf{r}, \omega)$ induced by an external potential $V^{e x t}(\mathbf{r}, \omega)$ is given by (atomic units $\hbar=m=e=1$ are used unless specified otherwise)

$$
\begin{aligned}
\delta \rho(\mathbf{r}, \omega) & =\int \chi\left(\mathbf{r}, \mathbf{r}^{\prime}, \omega\right) V^{e x t}\left(\mathbf{r}^{\prime}, \omega\right) d^{3} \mathbf{r}^{\prime} \\
& =\int \chi^{\mathrm{IPA}}\left(\mathbf{r}, \mathbf{r}^{\prime}, \omega\right) V^{\mathrm{SCF}}\left(\mathbf{r}^{\prime}, \omega\right) d \mathbf{r}^{\prime},
\end{aligned}
$$

where $\chi\left(\mathbf{r}, \mathbf{r}^{\prime}, \omega\right)$ is a full dynamical susceptibility or a response function, $\chi^{\mathrm{IPA}}\left(\mathbf{r}, \mathbf{r}^{\prime}, \omega\right)$ is a dynamical susceptibility under the independent-particle approximation, and $V^{\mathrm{SCF}}(\mathbf{r}, \omega)$ is the self-consistent field including both the external field and the field induced by the density change.

We use Eq. (2) to calculate the density changes instead of using Eq. (1) since it is difficult to calculate the full dynamical susceptibility. The susceptibility $\chi^{\mathrm{IPA}}\left(\mathbf{r}, \mathbf{r}^{\prime}, \omega\right)$ is written as

$$
\chi^{\mathrm{IPA}}\left(\mathbf{r}, \mathbf{r}^{\prime}, \omega\right)=\sum_{i, j}\left(n_{i}-n_{j}\right) \frac{\psi_{i}^{*}(\mathbf{r}) \psi_{j}(\mathbf{r}) \psi_{i}\left(\mathbf{r}^{\prime}\right) \psi_{j}^{*}\left(\mathbf{r}^{\prime}\right)}{\omega-\left(\epsilon_{j}-\epsilon_{j}\right)+i \eta},
$$

with $\eta$ a positive infinitesimal used to ensure the outgoing wave boundary conditions. $\left\{\psi_{i}(\mathbf{r})\right\}$ and $\left\{\epsilon_{i}\right\}$ are the eigenfunctions and eigenenergies of the single electron orbitals and $n_{i}$ is the occupation number for a given orbital. $\left\{\psi_{i}(\mathbf{r})\right\}$ and $\left\{\boldsymbol{\epsilon}_{i}\right\}$ are calculated from the Schrödinger equation

$$
\left[-\frac{1}{2} \nabla^{2}+V^{e f f}(r)\right] \psi_{i}(\mathbf{r})=\epsilon_{i} \psi_{i}(\mathbf{r})
$$

where $V^{e f f}(r)$ is an effective potential obtained from the optimized effective potential method [25]. The $V^{\mathrm{SCF}}(\mathbf{r}, \omega)$ satisfies the equation

$$
\begin{aligned}
V^{\mathrm{SCF}}(\mathbf{r}, \omega)= & V^{e x t}(\mathbf{r}, \omega)+\int \frac{\delta \rho(\mathbf{r}, \omega)}{\left|\mathbf{r}-\mathbf{r}^{\prime}\right|} d \mathbf{r}^{\prime} \\
& +\left.\frac{\partial V_{x c}(\mathbf{r})}{\partial \rho(\mathbf{r})}\right|_{\rho_{0}(\mathbf{r})} \delta \rho(\mathbf{r}, \omega),
\end{aligned}
$$

where $V_{x c}(\mathbf{r})$ is the exchange-correlation potential and $\rho_{0}(\mathbf{r})$ is the ground-state electron density.

For the electron-impact excitation or ionization under the Born-approximation, the external field is

$$
V^{e x t}(\mathbf{r}, \omega)=\exp [i \mathbf{K} \cdot \mathbf{r}]
$$

and the generalized oscillator strength (GOS) is written as

$$
f(\omega, K)=\frac{2 \omega}{K^{2}}\left|\left\langle\Psi_{f}\left|\sum_{j} \exp \left[i \mathbf{K} \cdot \mathbf{r}_{j}\right]\right| \Psi_{0}\right\rangle\right|^{2} \delta\left(E_{f}-E_{0}-\omega\right) .
$$

In the equation above, $\Psi_{0}$ and $\Psi_{f}$ are the initial and final states of the target atom with energies $E_{0}$ and $E_{f}, \omega$ and $\mathbf{K}$ are the impact electron energy loss and momentum transfer during the collision, and the summation $j$ runs over all the electrons of the target atom.

Using the electron-density change $\delta \rho(\mathbf{r}, \omega)$ due to $V^{\text {ext }}(\mathbf{r}, \omega)$, the GOS is expressed as

$$
f(\omega, K)=\frac{2 \omega}{K^{2}} \operatorname{Im} \int V^{e x t}(\mathbf{r}, \omega) \delta \rho(\mathbf{r}, \omega) d \mathbf{r} .
$$

Due to parity conservation and angular-momentum conservation, we can decompose each $\ell$ th pole contribution by replacing $V^{\text {ext }}(\mathbf{r}, \omega)$ with

$$
V_{\ell}^{e x t}(\mathbf{r}, \omega)=\sum_{m} 4 \pi i^{\ell} j_{\ell}(K r) Y_{\ell m}^{*}(\hat{\mathbf{K}}) Y_{\ell m}(\hat{\mathbf{r}})
$$

in Eqs. (2) and (5) and obtain the corresponding partial GOS labeled as $f^{\ell}(\omega, K)$. Thus, the total GOS can be rewritten as

$$
f(\omega, K)=\sum_{\ell} f^{\ell}(\omega, K) .
$$

With the calculated GOS, the electron-impact excitation or ionization cross sections are obtained as

$$
\sigma(\omega)=\frac{2 \pi}{E_{e}} \frac{1}{\omega} \int_{K_{\min }}^{K_{\max }} \frac{f(\omega, K)}{K} d \mathbf{K},
$$

with $E_{e}$ the incident electron kinetic energy and $K_{\min }\left(K_{\max }\right)$ the minimum (maximum) momentum transfer.

The differential cross section of an electron with energy loss $\omega$ at scattering angle $\Omega$ is given by

$$
\frac{d \sigma(\omega)}{d \Omega}=\frac{d \sigma(\omega)}{d \hat{K}} \frac{d \hat{K}}{d \Omega} \propto \sum_{\ell} f^{\ell}(\omega, K) .
$$

The scattering angle $\theta$ and the momentum transfer $K$ are related by the following equation as

$$
K^{2}=k_{i}^{2}+k_{f}^{2}-2 k_{i} k_{f} \cos \theta,
$$

with $k_{i}, k_{f}$ are the incident electron momenta before and after the collision. The equations derived above are for a paired spin system, but these equations can be extended to the case of an unpaired spin system in a straightforward manner [26].

\section{RESULTS AND DISCUSSION}

Using Eq. (12), we calculated the impact electron energyloss spectra when argon's $3 s$ electron is excited to some resonant states or its $3 p$ electron is ionized. The energy-loss spectra from 24.5 to $30.0 \mathrm{eV}$ were calculated in $0.01 \mathrm{eV}$ intervals. In the present calculation, we took into account monopole, dipole, and quadrupole contributions, but ignored higher multipole contributions because their effects are negligible for an incident energy of $200 \mathrm{eV}$. Since we already showed in 

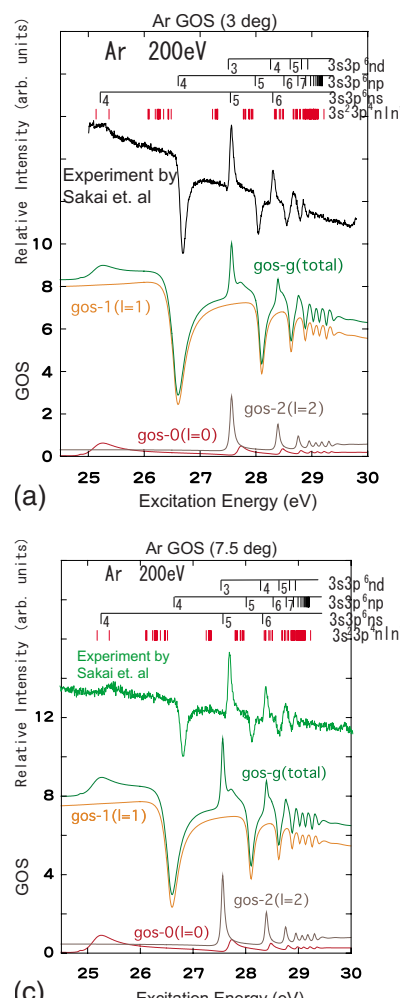

(c)

FIG. 1. (Color online) The calculated generalized oscillator strengths as a function of the impact electron energy loss using Born approximation in the case of $200 \mathrm{eV}$ impact energy at different scattering angles: (a) $3^{\circ}$, (b) $5^{\circ}$, (c) $7.5^{\circ}$, and (d) $10^{\circ}$. The label gos- $\ell$ refers to the partial GOS with $\ell$ pole, i.e.. Also, gos- 0 refers to the monopole term, gos- 1 to the dipole term, and gos- 2 to the quadrupole term. The label gos-g refers to the total GOS. Experimental spectra by Sakai et al. [21] are also plotted. (Regarding the scales of coordinates, GOS is used for theoretical values given in dimensionless number and "relative intensity" is used for experimental values)

an earlier paper [16] that for small scattering angles $(\theta$ $\leq 3^{\circ}$ ) the spectra are dominated by optically allowed transitions, in this paper we focused on the corresponding spectra for large scattering angles $\theta \geq 3^{\circ}$, which involve optically forbidden transitions. We compared our calculations to recent experimental measurements [21]. It should be noted that the calculated energy-loss spectra are shifted by $-0.43 \mathrm{eV}$ in order to match the $3 s 3 p^{6} 4 p$ peak from the measured photoabsorption spectra [6].

Figure 1 shows the calculated energy-loss spectra (GOS) with different scattering angles with $200 \mathrm{eV}$ incident energy for $\ell=0,1,2$ and the total GOS. The experimental results by Sakai et al. are also plotted. Let us first focus on Fig. 1(a). The GOS for $\ell=1$ (the dipole term) shows a continuum spectrum with several window resonances. As shown in our earlier paper, this is the same as the oscillator strength of the photoabsorption measured by Madden et al. [6]. These window resonances are attributed to transitions from the ground state to $3 s 3 p^{6} n p$ states and are very similar to the opticalabsorption spectra. The spectra of the electron energy loss are broader than the optical-absorption spectra. Apart from the dipole contributions, quadrupole and monopole transi- tions also exist. The contribution of these background continuum spectra to the total spectrum is much smaller than the corresponding contribution of the dipole background spectrum, which means that ionization from the $3 p$ valence orbital to the continuum states occurs through dipole-allowed transitions rather than dipole-forbidden transitions.

For $\ell=2$, ionization occurs from the ground state to continuum states with a total angular momentum of $L=2$. These continuum states $\left(3 s^{2} 3 p^{5} \epsilon p\right.$ or $\left.\epsilon f^{1} D\right)$ are coupled with the discrete $L=23 s 6 p^{6} n d{ }^{1} D$ states. The excitations from the ground state to the $3 s 6 p^{6} n d^{1} D$ states appear as peak type resonances. For $\ell=0$, ionization to $3 s^{2} 3 p^{5} \epsilon p{ }^{1} S$ occurs less than that for the case $\ell=2$. The same holds true for excitations to $3 s 3 p^{6} n s{ }^{1} S$.

The total GOS spectrum is shown with a lineog gos-g (total), which corresponds to the experimental observations. We see that the window resonance lines of $3 s 3 p^{6} n p,{ }^{1} \mathrm{P}$, the peak lines of $3 s 3 p^{6} n d^{1} D$, and the small bumps of $3 s 3 p^{6} n s{ }^{1} S$ are all well reproduced by our calculations. We also see that the background resulting from ionization to the $3 s^{2} 3 p^{5} \epsilon s+3 s^{2} 3 p^{5} \epsilon d$ states decreases with the excitation energy more slowly than experimental observations suggest. This may be due to the dependence of the measuring apparatus's electron collection efficiency on the electron energy.

The energy levels of $3 d$ and $5 s$ states are very close to each other, which is also the case for the $4 d$ and $6 s$ states. It is difficult to assign the experimental peaks only from their energy positions. In our calculations, we decompose the contributions into partial GOSs as shown in Fig. 1. Comparing to the experimental spectra, we assigned the peak around $27.5 \mathrm{eV}$ to the $3 s 3 p^{6} 3 d$ state and the peak around $28.5 \mathrm{eV}$ to the $3 s 3 p^{6} 4 d$ state.

As shown in Figs. 1(a)-1(d), as the scattering angle increases, the contributions from optically allowed transitions decrease and contributions from optical forbidden transitions gradually increase. The window resonances become shallow gradually as the scattering angle increases.

What happens if the scattering angle becomes large? Since there are no experimental data for large scattering angles, we only show our calculated energy-loss spectra for the scattering angles of $20^{\circ}, 30^{\circ}, 45^{\circ}$, and $60^{\circ}$ in Fig. 2 . Up to $30^{\circ}$, the dipole transition $(\ell=1)$ is the main contribution to the background ionization spectra. For larger scattering angles $\theta \geq 45^{\circ}$, the quadrupole transition becomes the main transition channel and all the resonance lines become peak type. For large scattering angles or large momentum transfers, the background ionization continuum becomes small for optically allowed transition in comparison to the excitation cross sections. Consequently, the window resonances due to the optically allowed transitions change into peaks. It should also be noted that the absolute value of the GOS decreases dramatically as the scattering angle or the momentum transfer increases.

\section{FANO'S PROFILE}

To discuss the change of resonance profile, it is convenient to use what is called the $q$ parameter-a parameter introduced by Fano [1]. To understand the meaning of this 

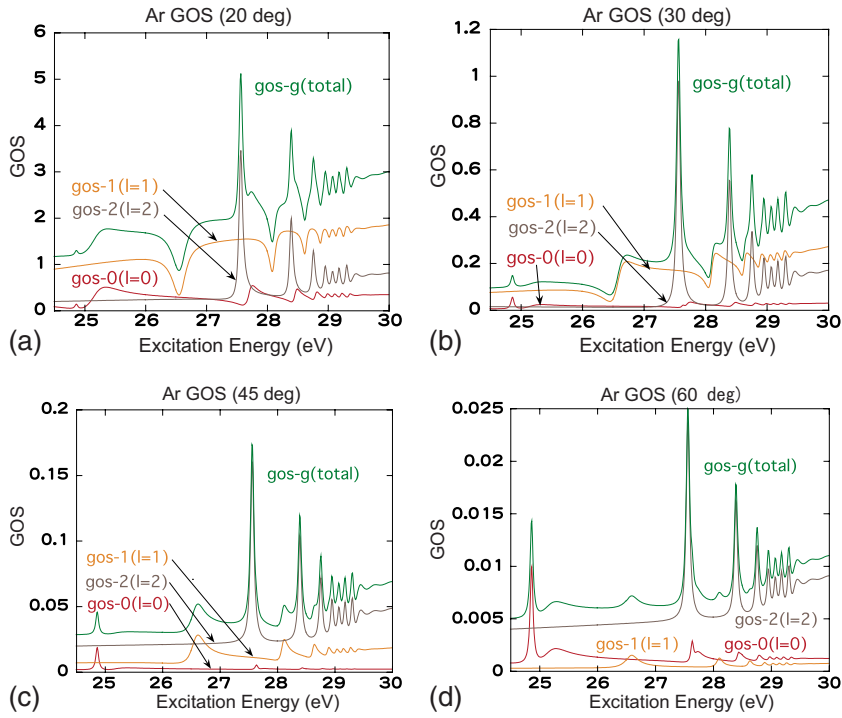

FIG. 2. (Color online) The same as Fig. 1 for the large scattering angles as (a) $\theta=20^{\circ}$, (b) $\theta=30^{\circ}$, (c) $\theta=45^{\circ}$, and (d) $\theta=60^{\circ}$.

parameter, we must note that the resonance line profile, the absorption cross section per unit energy $d \sigma / d E$ which is embedded in the ionization continuum $f_{c}(E)$, can be expressed in terms of excitation energy $E$, the energy of resonant level $E_{r}$, and resonance line width $\Gamma$ as

$$
\frac{d \sigma}{d E}=f_{a}\left(\frac{(q+\epsilon)^{2}}{1+\epsilon^{2}}-1\right)+f_{c}(E)
$$

where

$$
\epsilon=\frac{E-E_{r}}{\Gamma / 2}
$$

In short, Fano's $q$ parameter characterizes the line shape of the resonant absorption.

We fit the calculated GOS to the above formula to obtain the $q$ values. Figure 3 shows the $q$ values as a function of $K^{2}$, the square of the momentum transfer, for optically allowed transitions to the $3 s 3 p^{6} 3 p{ }^{1} P$ state [Fig. 3(a)] and optically forbidden transitions to the $3 s 3 p^{6} 3 d^{1} D$ state. Overall, our calculated $q$ values are in reasonable agreement with the corresponding values computed experimental measurements.
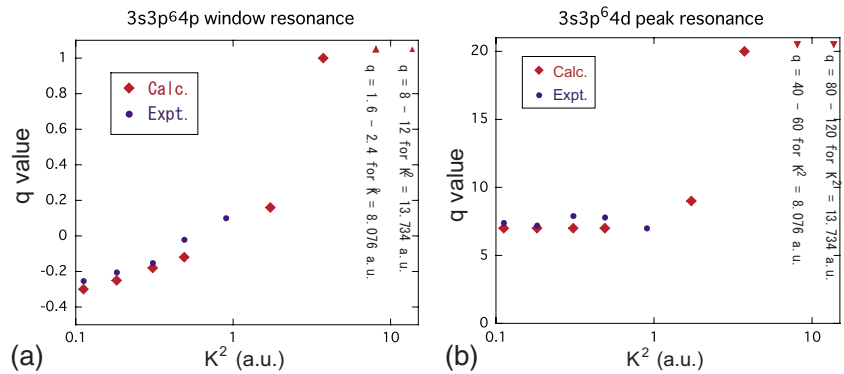

FIG. 3. (Color online) The $q$ parameter of $3 s 3 p^{6} n p$ window resonance (left panel) and $2 s 3 p^{6} n d$ sharp resonance (right panel) as a function of $K^{2}$ in logarithmic scale. Experimental values are by Sakai et al. [21] and Zhu et al. [27].
For optically allowed transitions, the $q$ value starts from a negative value and increases as $K^{2}$ increases. The $q$ value reaches zero at $K^{2} \cong 0.2$. The positive and negative $q$ values indicate different asymmetrical line profiles. To study the physical origins of the line profile changes, let us go back to the definition of the $q$ value. The $q$ value is defined as

$$
q=\frac{\left\langle\psi_{i}|T| \psi_{r}\right\rangle}{\left\langle\psi_{i}|T| \psi_{c}\right\rangle},
$$

where $\psi_{i}$ is the initial-state wave function and $\psi_{r}$ and $\psi_{c}$ the wave functions of the resonant state and the final continuum, respectively. In general, $T$ may be any kind of electronic transition operator. For the present case, however, $T$ is approximated as $\exp [i \mathbf{K} \cdot \mathbf{r}] /|\mathbf{K}|$, where $\mathbf{K}$ is momentum transferred to the scattered electron and $\mathbf{r}$ the electronic coordinate of the electron to be excited.

In 1980, Mitchell et al. [18] reported the GOSs of Ar, but they did not measure the spectra for the whole excitation energy region but instead measured precise spectra of resonance peaks and windows using electron-impact spectroscopy. They also measured the spectra impact energy dependence for small scattering angles and its angular scattering dependence for low-impact energies. Though they focused their attention on obtaining resonance profiles rather than the entire energy-loss spectra, we were able to compare their data results to our high-impact energy and small scattering angle results and determined that the two are consistent.

To compare the results of Mitchell et al. [18] to our results, we needed to obtain a relation between the parameter $D$ that Mitchell et al. introduced in their analysis and our calculated $q$ values. To obtain this relation, it is useful to introduce two new parameters $\eta$ and $\mu$ for $\epsilon$ and $q$ in Eq. (14), where $\epsilon=-\cot \eta(0 \leq \eta<\pi)$ and $q=-\cot \mu(0 \leq \mu$ $<\pi)$. With these new parameters, we can write

$$
\begin{gathered}
1+\epsilon^{2}=\frac{\sin ^{2} \eta+\cos ^{2} \eta}{\sin ^{2} \eta}=\frac{1}{\sin ^{2} \eta}, \\
(\epsilon+q)^{2}=(-\cot \eta-\cot \mu)^{2}=\frac{(\sin \eta \cos \mu+\cos \eta \sin \mu)^{2}}{(\sin \eta \sin \mu)^{2}} .
\end{gathered}
$$

It also follows that

$\frac{d \sigma}{d E}=f_{a}\left(\frac{1+\epsilon^{2}}{(\epsilon+q)^{2}}-1\right)+f_{c}(E)=f_{a}\left(\frac{\sin ^{2}(\eta+\mu)}{\sin ^{2} \mu}-1\right)+f_{c}(E)$.

As the energy $\epsilon$ changes from $-\infty$ to $\infty, \eta$ changes from 0 to $\pi$. This means that when $q=-\infty, \mu=0$, when $q=0, \mu=\pi / 2$, and when $q=+\infty, \mu=\pi$. Given a background wave function $\psi_{c}$ and a resonant state wave function $\psi_{r}$, a coupled state can be expressed as $\psi_{0}=\cos \eta \psi_{c}+\sin \eta \psi_{r}$. The total spectrum is given by $\left|\left\langle\psi_{0}|T| \psi_{f}\right\rangle\right|^{2} \propto \sin ^{2}(\eta+\mu)$.

This indicates that $\mu$ is the position when the interaction with the resonance level starts and that the interaction ends when $\eta=\pi$. The maximum value of the spectrum is $f_{a} /\left(1 / \sin ^{2} \eta-1\right)+f_{c}(E)$, occurs at $\eta+\mu=\pi / 2$, and the minimum value is $f_{c}(E)-f_{a}$, occurs at $\eta+\mu=0$ or $=\pi$. The value of $D$ which was defined by Mitchell et al. $\sin ^{2} \mu$ for $\pi / 2$ 


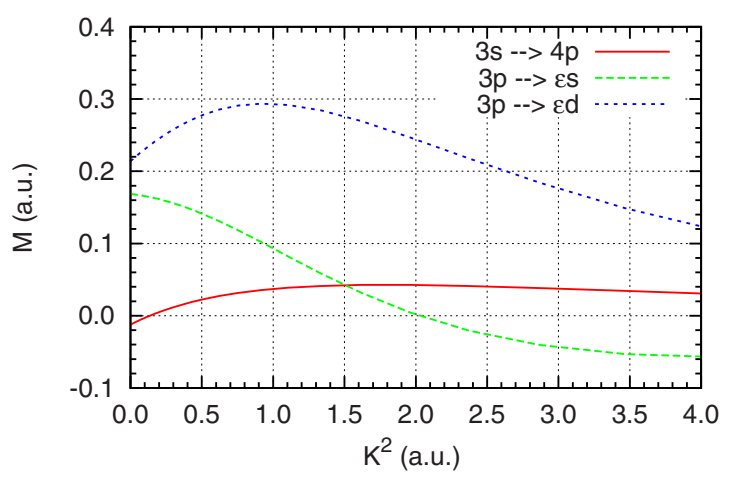

FIG. 4. (Color online) The transition matrix elements of $M$ $=\left\langle\psi_{i}\left|j_{1}(K r) Y_{1}(\hat{K} \hat{r})\right| \psi_{f}\right\rangle / K$ defined in Eq. (15) for the transitions of $3 s \rightarrow 4 p, 3 p \rightarrow \epsilon s$, and $3 p \rightarrow \epsilon d$.

$\leq \mu<\pi$. A variable $D$ is cut at $D= \pm 1(q=0)$ and in the present treatment, a branch cut is set at $\mu=n \pi(n=0,1)(q$ $=\mp \infty)$ as Fano defined $q$ in Ref. [1].

Figure 4 shows the matrix elements $M$ $=\left\langle\psi_{i}\left|j_{1}(K r) Y_{1}(\hat{K} \hat{r})\right| \psi_{f}\right\rangle / K$ as a function of $K^{2}$. The wave functions used in our calculation are obtained from Eq. (4). We see that the $3 s \rightarrow 4 p$ matrix element changes its sign around $K^{2} \cong 0.2$. This means that a sign change in the $q$ value is associated with change in shape of the resonance structure. For example, as the $q$ value changes from negative to positive, a resonance structure having a profile similar to a sine function over one full cycle (0 to $2 \pi$ ) will change to the structure having a profile similar to the sine function reflected about the $x$ axis. In other words, as the $q$ value changes from negative to positive, the positive hump of the profile will become a negative hump and vice versa. Admittedly, this is only a qualitative observation, but it does provide some insight into the physical meaning of the $q$ value. It should be noted that the resonant state interacts with two continuum states. Although the transition matrix of $3 p \rightarrow \epsilon S$ changes its sign at $K^{2}=2.0$ while that of $3 p \rightarrow \epsilon d$ does not, the $q$ value does not undergo a sign change because the magnitude of the former transition matrix is small.

\section{SUMMARY AND CONCLUSIONS}

In the former paper [16], we studied the energy-loss spectra of charged particles incident on an Ar atom as a function of excitation energies for the case of small scattering angles (below $3^{\circ}$ ) by calculating the GOS using linear-response theory together with a optimized effective potential incorporating a self-interaction correction. In this paper, we extended the method to treat the case of larger electronscattering angles (from $3^{\circ}$ to $60^{\circ}$ ) with an impact energy of $200 \mathrm{eV}$. The results of our calculations showed good agreement with the results of recent experiments. Finally, it should be noted that the data calculated for a particle having a given incident energy and a large scattering angle can be compared directly to a case having a higher incident energy and smaller scattering angle. This is because Eq. (11) does not depend on the electron incident energy explicitly. In other words, our results can be used to study cases where the incident particle's energies are much larger than $200 \mathrm{eV}$. For example, the case of an electron having an incident energy of $200 \mathrm{eV}$ and a scattering angle of $60^{\circ}\left(K^{2}=13.7\right.$ a.u. $)$ corresponds to the case in which an electron has an incident energy of $200 \mathrm{KeV}$ and a scattering angle of $2.5^{\circ}$.

\section{ACKNOWLEDGMENTS}

This research was partially supported by Grand-in-Aid for Scientific Research (B) and (C) from the Japan Society for the Promotion of Science. The authors would like to express sincere thanks to Professor S. Ohtani, Professor C. Yamada, and Professor N. Nakamura of the University of ElectroCommunications, Professor Y. Sakai of Toho University, and Professor T. Takayanagi of Sophia University for providing useful discussions.
[1] U. Fano, Phys. Rev. 124, 1866 (1961).

[2] J. A. Simpson, G. E. Chamberlain, and S. R. Mielczarek, Phys. Rev. 139, A1039 (1965).

[3] W. F. Chan, G. Cooper, and C. E. Brion, Phys. Rev. A 44, 186 (1991).

[4] S. J. Brotton, S. Cvejanovic, F. J. Currell, N. J. Bowring, and F. H. Read, Phys. Rev. A 55, 318 (1997).

[5] X. W. Fan and K. T. Leung, J. Phys. B 34, 811 (2001).

[6] R. P. Madden, D. L. Ederer, and K. Codling, Phys. Rev. 177, 136 (1969).

[7] B. W. Shore, Rev. Mod. Phys. 39, 439 (1967).

[8] F. H. Mies, Phys. Rev. 175, 164 (1968).

[9] P. G. Burke and D. D. McVicar, Proc. Phys. Soc. 86, 989 (1965).

[10] M. Venuti, P. Decleva, and A. Lisini, J. Phys. B 29, 5315 (1996).

[11] T. Morishita, K. I. Hino, S. Watanabe, and M. Matsuzawa,
Phys. Rev. A 53, 2345 (1996).

[12] S. Salomonson, S. L. Carter, and H. P. Kelly, Phys. Rev. A 39, 5111 (1989).

[13] T. Schneider, C. N. Liu, and J. M. Rost, Phys. Rev. A 65, 042715 (2002).

[14] Z. S. Yuan, X. Y. Han, X. J. Liu, L. F. Zhu, K. Z. Xu, L. Voky, and J. M. Li, Phys. Rev. A 70, 062706 (2004).

[15] C.-N. Liu, A.-T. Le, T. Morishita, B. D. Esry, and C. D. Lin, Phys. Rev. A 67, 052705 (2003).

[16] X. M. Tong and T. Watanabe, Phys. Rev. A 76, 042715 (2007).

[17] P. G. Burke and K. T. Taylor, J. Phys. B 8, 2620 (1975).

[18] P. Mitchell, J. A. Baxter, J. Comer, and P. J. Hicks, J. Phys. B 13, 4481 (1980).

[19] S. L. Wu, Z. P. Zhong, R. F. Feng, S. L. Xing, B. X. Yang, and K. Z. Xu, Phys. Rev. A 51, 4494 (1995).

[20] P. Mitchell, D. L. Ederer, and J. Comer, J. Phys. B 13, 2817 (1980). 
[21] Y. Sakai et al. (unpublished).

[22] R. Kubo, J. Phys. Soc. Jpn. 12, 570 (1957).

[23] R. G. Parr and W. T. Yang, Density-Functional Theory of Atoms and Molecules (Oxford University Press, New York, 1989).

[24] A. Fetter and J. Walecka, Quantum Theory of Many-Body Sys- tems (McGraw-Hill, New York, 1971).

[25] X. M. Tong and S. I. Chu, Phys. Rev. A 55, 3406 (1997).

[26] X. M. Tong, D. Kato, T. Watanabe, and S. Ohtani, J. Phys. B 33, 717 (2000).

[27] L. F. Zhu, H. D. Cheng, X. J. Liu, P. Tian, Z. S. Yuan, W. B. Li, and K. Z. Xu, Chin. Phys. Lett. 20, 1718 (2003). 